\title{
EFFECTIVENESS OF PATIENT-SPECIFIC THORACOLUMBAR BRACE TREATMENT FOR ADOLESCENT IDIOPATHIC SCOLIOSIS: A PROSPECTIVE COHORT STUDY
}

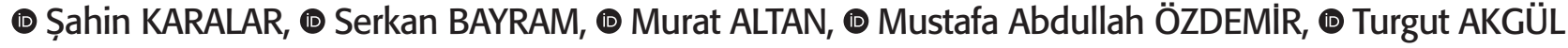 \\ Istanbul University, Istanbul Faculty of Medicine, Department of Orthopaedics and Traumatology, Istanbul, Turkey
}

\begin{abstract}
Objective: The efficacy of bracing for patients with adolescent idiopathic scoliosis (AIS) remains controversial. We aimed to evaluate the effectiveness of patient-specific thoracolumbar brace treatment in patients with AIS who met the Scoliosis Research Society inclusion criteria and the factors affecting success rate.

Materials and Methods: From April 2015 to February 2018, 25 patients diagnosed with AIS treated with patient-specific thoracolumbar brace were asked to participate in this study. The initial brace correction rate and clinical outcomes of the main curvature was evaluated. The clinical course of bracing was considered progression if there was $\geqslant 6^{\circ}$ curvature increase and improvement if there was $\geqslant 6^{\circ}$ curvature decrease. The success rate was correlated with age, sex, Lenke classification, Risser grade, initial Cobb angle and rotation grade.

Results: The curvature progressed and improved in 13 and two cases, respectively, and the curve remained unchanged in 10 cases. A success rate of $48 \%(12 / 25)$ was achieved. Moreover, only three of 25 cases with Cobb angle of $45^{\circ}$ were considered candidates for surgery. The mean prebrace Cobb angle of the main curvature was $27.9^{\circ} \pm 6.7^{\circ}$, which ranged from $20^{\circ}$ to $37^{\circ}$. The duration of bracing was $37.2(6-76)$ months. The mean Cobb angle at the end of the treatment was $32.1^{\circ} \pm 8.2^{\circ}$, which ranged from $15^{\circ}$ to $45^{\circ}$. No correlation was found between age, Risser grade and brace treatment success. However, treatment success was significantly correlated with initial Cobb angle, rotation grade and Lenke classification (main thoracic) ( $p<0.001, r=0.680 ; p=0.028, r=-0.458 ; p=0.020, r=0.481$, respectively).

Conclusion: Patient's age, Risser grade and sex were not related with successful results of brace treatment. However, the initial Cobb angle, rotation of the apical vertebra and Lenke classification were significantly correlated with successful brace treatment.

Keywords: Brace treatment, patient-specific thoracolumbar brace, adolescent idiopathic scoliosis, conservative treatment
\end{abstract}

\section{INTRODUCTION}

The treatment for scoliosis remains debatable, especially for patients with adolescent idiopathic scoliosis (AIS) with Cobb angle of $10^{\circ}-25^{\circ}$. Rehabilitative activity and bracing are accepted in various studies as conservative treatment protocol ${ }^{(1-3)}$. Bracing is initiated for patients with curvature of $20^{\circ}-30^{\circ}$ and $\geqslant 5^{\circ}$ improvement of the curvature occurs during subsequent visits. Notwithstanding, when a patient is skeletally immature (Risser grade $\leqslant 2$ ) and presented with a $30^{\circ}-45^{\circ}$ curve, bracing is suggested at the first visit( ${ }^{(4)}$. At the Osaka Medical College, several braces have been recommended, such as Boston, Milwaukee, Wilmington, soft braces (SpineCor/ TriaC) and night-time braces (Providence/Charleston) $)^{(3)}$. The implementation of orthopaedic braces could be propitious for restraining curvature progression in patients with $\mathrm{AIS}^{(5)}$. As regards bracing, the percentage of in-brace correction and brace wearing time can affect the outcome of bracing(6). Each brace should be assessed separately because various kinds of braces yield different results ${ }^{(7,8)}$. It was thought that brace treatment success is related to patients' compliance and usage time. In the literature, patient-specific thoracolumbar brace was reported to have satisfying outcomes. However, the success rate depends on the patients' compliance. In this study, we aimed to evaluate the effectiveness of the patient-specific thoracolumbar brace treatment in patients with AIS and the factors influencing the success rate.

\section{MATERIALS AND METHODS}

This prospective therapeutic study was conducted at our clinic from April 2016 to February 2018. Patients with diagnosis of progressing idiopathic scoliosis were asked to participate in this study. Ethics committee approval was acquired for this research from the ethics board of İstanbul University, İstanbul Faculty of Medicine (2018/1500). Written informed consent was obtained from patients and parents of children who participated in

Address for Correspondence: Serkan Bayram, İstanbul University, İstanbul Faculty of Medicine, Department of Orthopaedics and Traumatology, İstanbul, Turkey E-mail: dr.serkanbayram89@gmail.com Received: 15.10.2020 Accepted: 27.10.2020

ORCID ID: orcid.org/0000-0001-7651-1200 
turkishspine

this study. A prospective cohort study was then performed to evaluate the effectiveness of patient-specific thoracolumbar brace in the treatment of AIS.

The inclusion criteria were as follows: patients who met the Scoliosis Research Society (SRS) inclusion criteria which consist of age $\geqslant 10$ years upon prescription of the brace, Risser $0-2$, primary curve angles of $25^{\circ}-40^{\circ}$, no prior treatment and, if female, either premenarche or less than 1 year postmenarche ${ }^{(9)}$, and a minimum of 2 years follow-up.

The exclusion criteria were history of brace treatment and comorbidities that change the course of AIS such as genetic defects, neuromuscular disorders, metabolic disorders, and history of severe trauma.

Patient-specific thoracolumbar braces were fabricated, and functions that consisted of pressure pad placements were ensured by the same certified orthotist. A plaster cast was taken to capture the body shape of each patient with an underarm position. In addition, standing anteroposterior (AP) X-ray images were used to confirm in-brace correction and full spinal alignment, including the pelvis, while the brace was being worn (Figure 1). The correction magnitude threshold was $>50 \%$ reduction of the initial Cobb angle. At the beginning of bracing, patients were instructed to wear the brace for a minimum of 23 hours per day. Bracing was stopped at 1 year after skeletal maturity was reached. Skeletal maturity was achieved if all of the following three criteria were fulfilled: Risser stage 4, at least 2 years after the onset of menstruation (for girls) and two consecutive visits for at least 1 year with no more than a $1-\mathrm{cm}$ increase in height.

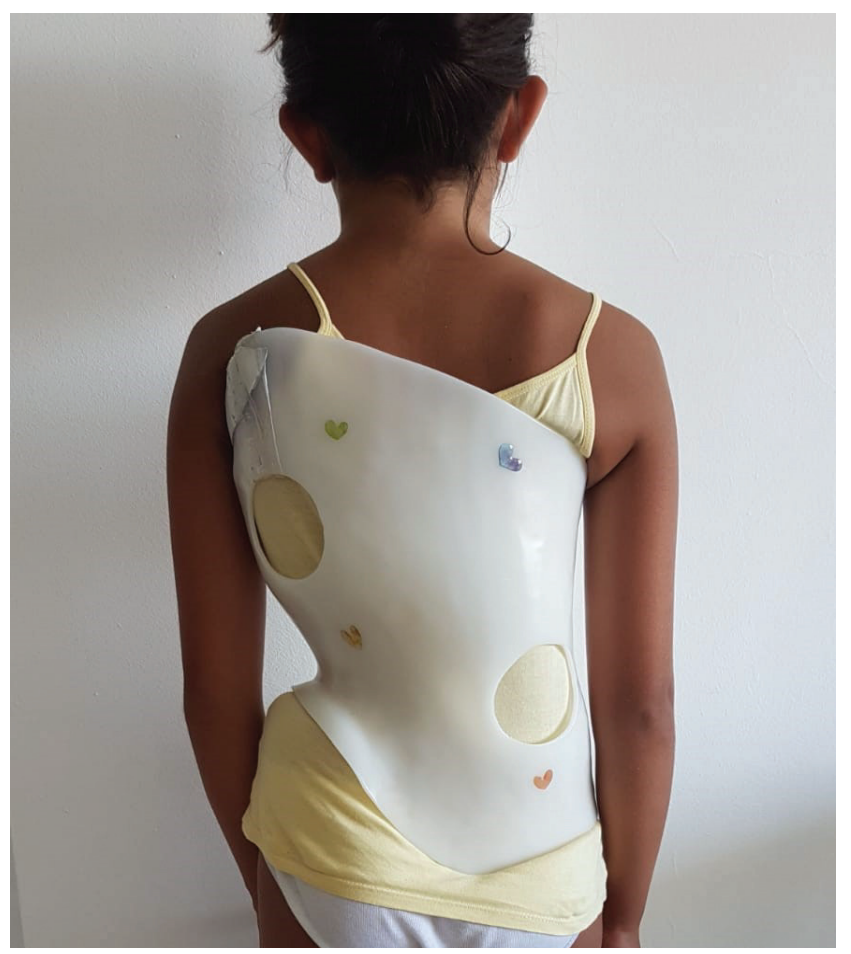

Figure 1. The photo was showed that the patient treated with patient specific thoracolumbar brace
At the beginning of the treatment, 25 patients with AIS (22 girls and three boys) aged $11.4 \pm 1.19$ years (range: $10-14$ ) were analysed. X-ray imaging was done before the start of treatment, while the brace was worn, before and after each use of subsequent braces and at skeletal maturity (after brace wearing). In-brace X-ray images were taken 6 weeks after the start and 6 months after the bracing period to determine 'skeletal maturity' values. All Cobb angle analyses were done by the senior author (TA). After combining curve types into main thoracic and main lumbar, 10 patients were considered to have main thoracic curves (Lenke I, II, or III), and the remaining 15 patients were ascertained to have main lumbar curves (Lenke V or VI). The rotation of the apical vertebra was also measured using the Nash and Moe method, which was based on the relationship between the vertebral pedicles and the centre of the vertebral body in the AP X-ray view ${ }^{(10)}$. Rotation was classified in five degrees according to the removal of the pedicles. No vertebral rotation was identified if the pedicles are halfway to the lateral margins of the vertebral bodies and considered at $0^{\circ}$. As the projection of the pedicle of the apical vertebra moves towards the median line in the AP view, the rotational degree progresses in the evaluation scale, reaching the most significant value (degree IV) when it crosses that line. The clinical outcome was assessed based on the SRS criteria. According to the Cobb angle on standing AP spine X-ray images, which obtained with the patients not wearing the brace, were classified as follows: (1) improved, decrease in the Cobb angle by $\geqslant 6^{\circ}$; (2) stable, no more than $5^{\circ}$ of progression or improvement; (3) progressed, increase in the Cobb angle by $\geqslant 6^{\circ}$ and (4) progression with Cobb angle $\geqslant 45^{\circ}$ was considered candidates for surgery.

\section{Statistical Analysis}

Statistical analysis was conducted using the SPSS statistics version 24.0 (IBM Corp., Armonk, NY). Descriptive statistics (mean, standard deviation, median, frequency, ratio, minimum and maximum) were used to evaluate the study data. Student's t-test was used to differentiate two groups of quantitative data with normal distribution, and the Mann-Whitney $U$ test was used to compare two groups of data with non-normal distribution. Pearson chi-square test, Fisher-Freeman-Halton exact test and Fisher's exact test were used to compare qualitative data; with a significance level set a priori at $p<0.05 \mathrm{~A} p$ value less than 0.05 was deemed to be statistically notable.

\section{RESULTS}

The first series involved 25 patients (three boys, 22 girls). Three patients underwent scoliosis surgery. The curvature type were main thoracic $(n=8)$, thoracolumbar $(n=10)$, lumbar $(n=5)$, double major $(n=1)$ and double thoracic $(n=1)$. Risser stage was grade 1 in eight, grade 2 in eight and grade 2-3 in nine cases. The apexes of the main curves were higher than $\mathrm{T} 7$ in four patients (T6 in four patients) and lower than T7 in 11 patients (T8 in four, T9 in three, T10 in one, T11 in one, T12 in two, L1 in two, L2 
in five and L3 in three cases). The mean pre-brace Cobb angle of the main curves was $27.9^{\circ} \pm 6.7^{\circ}$, which ranged from $20^{\circ}$ to $37^{\circ}$. The duration of bracing was 37.2 (range: 16-76) months. The mean Cobb angle at end of the treatment was $32.1^{\circ} \pm 8.2^{\circ}$, which ranged from $15^{\circ}$ to $45^{\circ}$. According to Nash and Moe classification, 12 patients had grade 1 , six had grade 2, two had grade 3 and four had grade 4 rotation.

At the last follow-up, the curve progressed in 13 cases, improved in two cases and remained unchanged in 10 cases (Figure 2 A, B and Figure 3 A, B). A success rate of 48\% (12/25) was accomplished. Furthermore, only three of 25 cases that progressed beyond Cobb angle of $45^{\circ}$ were admitted as candidates for surgery.

No correlation was found between age, Risser grade and thriving brace treatment outcome. However, successful treatment was significantly associated with the initial Cobb angle, rotation grade and Lenke classification (main thoracic) $(p<0.001, r=0.680$; $p=0.028, r=-0.458 ; p=0.020, r=0.481$, respectively).

\section{DISCUSSION}

In this study, we used age and simple morphologic classifications (Cobb, Lenke classification and Risser grade) and demonstrated successful treatment of AIS using patientspecific thoracolumbar brace and the relationship between

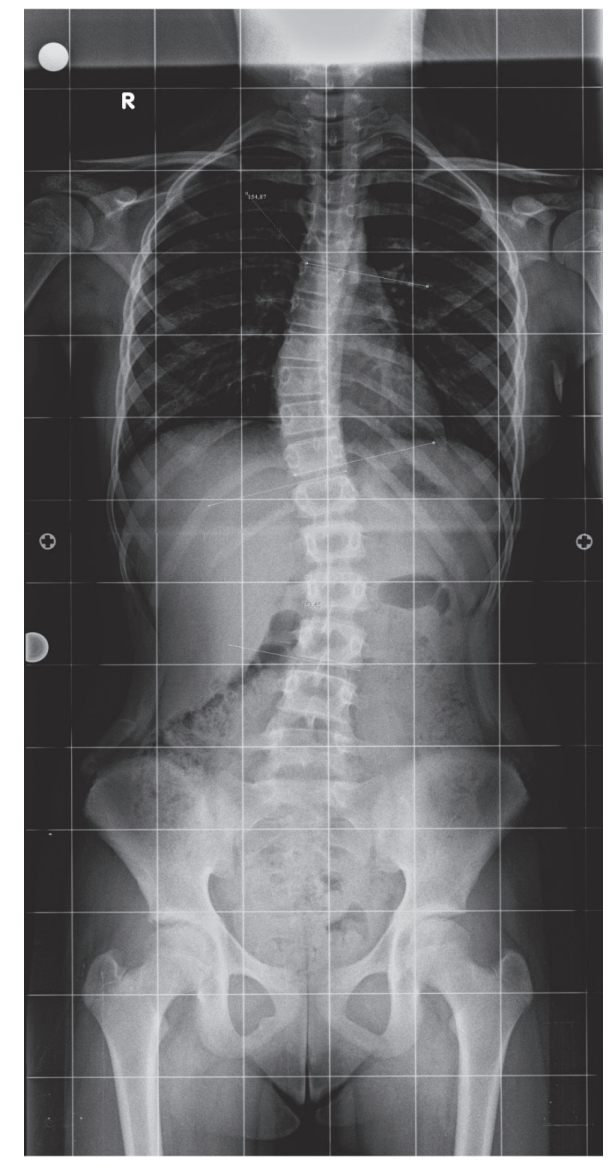

Figure 2A. Standing anteroposterior radiograph of the patient before beginning of the patient specific thoracolumbar brace uncomplicated parameters and brace benefit. Thompson et al. ${ }^{(11)}$ reported a comprehensive series managed with thoracolumbosacral orthosis brace in 168 patients. In their study, the rate of surgery or improvement to $\geqslant 50^{\circ}$ was $35.8 \%(43$ of 120 ) in patients with persistent main thoracic curves, $20.0 \%$ (6 of 30 ) in patients with persistent main lumbar curves, $12.5 \%$ ( 1 of 8$)$ in patients with main thoracic curves that converted to main lumbar curves, and $0 \%(0$ of 9$)$ in patients with main lumbar curves that became main thoracic curves $(p=0.0383)$. The thoracic curves are at higher risk for brace failure than the lumbar curves regardless of the comparable primary curve magnitudes and average daily duration of wearing brace. Our study found a notable distinction between the main thoracic and main lumbar curves, that is, patients with thoracic curves had a higher success rate. We observed that rotation is also correlated with a thriving rate.

Previous clinical studies have highlighted that curve progression is associated with younger age ${ }^{(12,13)}$. Nevertheless, various studies have found no relationship between age and curve improvement. Cheung et al. ${ }^{(13)}$ presented a large series of 586 patients with mean brace-wear duration of $3.8 \pm 1.5$ years and a post-wearing follow-up duration of $2.0 \pm 1.1$ years. They found that curve progression was correlated with younger age [odds ratio (OR): 0.71 ( $95 \%$ confidence interval $(\mathrm{Cl}): 0.55$ to 0.91 ];

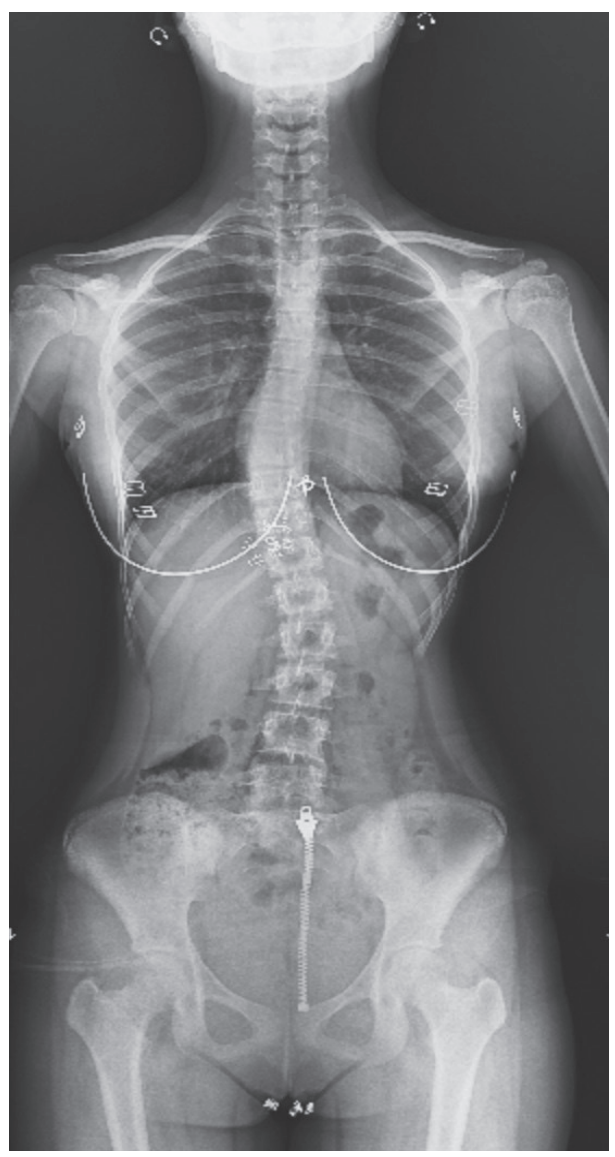

Figure 2B. The brace has been removed. The patient was successful treated with patient specific thoracolumbar brace 


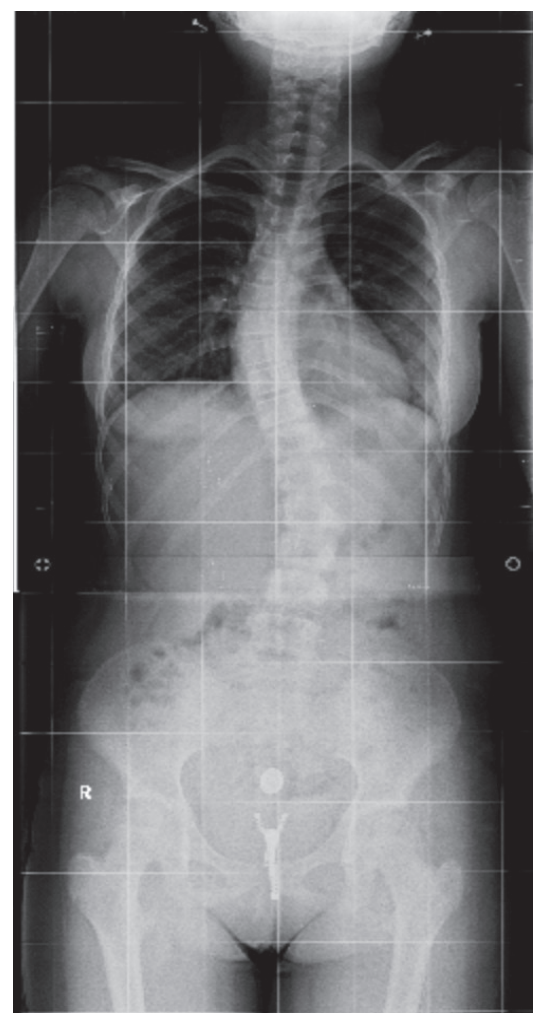

Figure 3A. Standing anteroposterior radiograph of the patient before beginning of the patient specific thoracolumbar brace

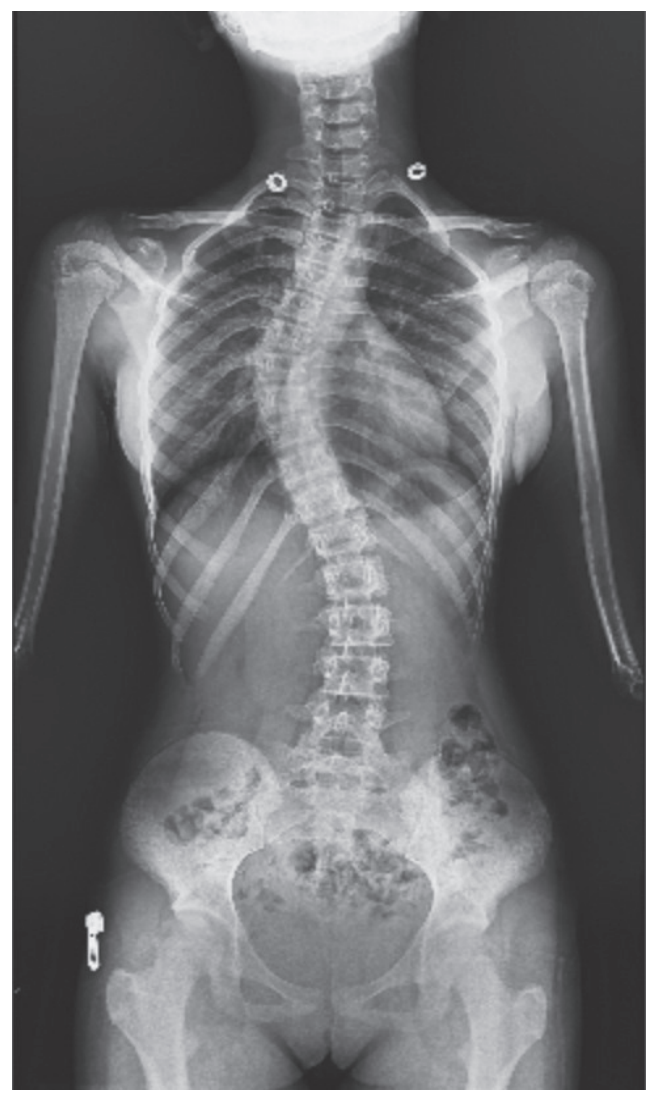

Figure 3B. The brace has been removed. The curve was progressed in this patient $p=0.008]$. Yrjönen et al. ${ }^{(14)}$ described 102 patients with AIS using Boston brace and reported that patient's age, curve pattern, or curve magnitude did not have a statistical influence on the risk of progression. Peltonen et al.(15) examined 107 patients with idiopathic scoliosis using Boston brace and average follow-up of 3 years. In their analysis, no correlation was found between patients' age at the start of the treatment and outcome. In our study, we have not obtained a relationship between age and curve increase.

Another main factor on the treatment AIS using brace is the initial curve. First, Emans et al.(16) advised that a Boston brace with a higher primary curve magnitude enhanced the potential for surgery. Katz and Durani et al.(17) found that double curves, with an initial thoracic curve $>35^{\circ}$, are more likely to progress. On the contrary, Ovadia et al. ${ }^{(18)}$ reported that low baseline Cobb angle values are linked to a more limited progression rate, but they did not find a statistically significant correlation. Kuroki et al. ${ }^{(19)}$ found that Cobb angles of $20^{\circ}-30^{\circ}$ were not significantly associated with lower success rate than angles $>30^{\circ}$, so they reported that curve magnitude is not associated with treatment success. Van den Bogaart et al. ${ }^{(20)}$ performed a systematic review of moderate scientific evidence that the initial Cobb angle was not related with treatment failure and inadequate evidence of treatment success. In the present study, we found a notable correlation between the primary Cobb angle and successful treatment.

\section{Study Limitations}

This study has several limitations. First, this study has a small sample size. Second, the follow-up time was relatively short. Third, the number of male patients was higher than that of female patients to correlate sex with brace success. Given our small sample size, we did not measure the Risser stage, which has been shown to influence brace success. Finally, the average hours of daily wearing of brace were not assessed, which was considered to influence brace compliance and success.

\section{CONCLUSION}

The patient-specific thoracolumbar brace treatment for AIS in skeletally immature patients could significantly decrease the increase in curve angle to the threshold for surgical intervention. Patient's age, Risser grade and sex were not related to optimum brace treatment outcomes. The initial Cobb angle, rotation of the apical vertebra and Lenke classification were significantly correlated with the spread of brace treatment.

\section{Ethics}

Ethics Committee Approval: Ethics committee approval was acquired for this research from the ethics board of İstanbul University, İstanbul Faculty of Medicine (2018/1500).

Informed Consent: Written informed consent was obtained from patients and parents of children who participated in this study Peer-review: Externally and internally peer-reviewed. 


\section{Authorship Contributions}

Concept: T.A., S.B., Design: S.B., Data Collection or Processing: Ş.K., T.A., Analysis or Interpretation: S.B., Literature Search: M.A., M.A.Ö., Writing: S.B., M.A.

Conflict of Interest: No conflict of interest was declared by the authors.

Financial Disclosure: The authors declared that this study received no financial support.

\section{REFERENCES}

1. Weiss HR, Goodall D. The treatment of adolescent idiopathic scoliosis (AIS) according to present evidence. A systematic review. Eur J Phys Rehabil Med. 2008;44:177-93.

2. Sy Ng, Bettany-Saltikov J, Moramarco M. Evidence for conservative treatment of adolescent idiopathic scoliosis - Update 2015 (MiniReview). Curr Pediatr Rev. 2016;12:6-11.

3. Negrini S, Minozzi S, Bettany-Saltikov J, Zaina F, Chockalingam N, Grivas TB, et al. Braces for idiopathic scoliosis in adolescents. Cochrane Database Syst Rev. 2010;1:CD006850.

4. Silva FE, Lenke LG. Adolescent idiopathic scoliosis. In: Errico TJ, Lonner BS, Moulton AW, eds. Surgical Management of Spinal Deformities. Philadelphia, Pa:Saunders Elsevier; 2009:97-118.

5. Fang MQ, Wang $C$, Xiang $\mathrm{GH}$, Lou $\mathrm{C}$, Tian NF, Xu HZ. Long-term effects of the Ch êneau brace on coronal and sagittal alignment in adolescent idiopathic scoliosis. J Neurosurg Spine. 2015;23:505-9.

6. Landauer F, Wimmer $\mathrm{C}$, Behensky $\mathrm{H}$. Estimating the final outcome of brace treatment for idiopathic thoracic scoliosis at 6-month followup. Pediatr Rehabil. 2003;6:201-7.

7. Guo J, Lam TP, Wong MS, Wah Ng BK, Lee KM, Liu KL, et al. A prospective randomized controlled study on the treatment outcome of SpineCor brace versus rigid brace for adolescent idiopathic scoliosis with follow-up according to the SRS standardized criteria. Eur Spine J. 2014;23:2650-7.

8. De Giorgi S, Piazzolla A, Tafuri S, Borracci C, Martucci A, De Giorgi G. Chêneau brace for adolescent idiopathic scoliosis: long-term results. Can it prevent surgery? Eur Spine J. 2013;22(Suppl6):S815-22.
9. Richards BS, Bernstein RM, D'Amato CR, Thompson $\mathrm{GH}$. Standardization of criteria for adolescent idiopathic scoliosis brace studies: SRS Committee on Bracing and Nonoperative Management. Spine (Phila Pa 1976). 2005;30:2068-75.

10. Nash CL, Moe JH. A study of vertebral rotation. J Bone Joint Surg Am. 1969;51:223-9.

11. Thompson RM, Hubbard EW, Jo CH, Virostek D, Karol LA. Brace success is related to curve type in patients with adolescent idiopathic scoliosis. J Bone Joint Surg Am. 2017;99:923-8.

12. Lonstein JE, Carlson JM. The prediction of curve progression in untreated idiopathic scoliosis during growth. J Bone Joint Surg Am. 1984;66:1061-71.

13. Cheung JPY, Cheung PWH, Yeng WC, Chan LCK. Does curve regression occur during underarm bracing in patients with adolescent idiopathic scoliosis? Clin Orthop Relat Res. 2020;478:334-45.

14. Yrjönen T, Ylikoski M, Schlenzka D, Poussa M. Results of brace treatment of adolescent idiopathic scoliosis in boys compared with girls: a retrospective study of 102 patients treated with the Boston brace. Eur Spine J. 2007;16:393-7.

15. Peltonen J, Poussa M, Ylikoski M. Three-year results of bracing in scoliosis. Acta Orthop Scand. 1988;59:487-90.

16. Emans ], Kaelin A, Bancel P, Hall J, Miller M. The Boston bracing system for idiopathic scoliosis: follow-up results in 295 patients. Spine. 1986;11:792-801.

17. Katz D, Durrani A. Factors that influence outcome in bracing large curves in patients with adolescent idiopathic scoliosis. Spine (Phila Pa 1976). 2001;26:2354-61.

18. Ovadia D, Eylon S, Mashiah A, Wientroub S, Lebel E. Factors associated with the success of the Rigo System Cheneau brace in treating mild to moderate adolescent idiopathic scoliosis. J Child Orthop. 2012;6:327-31.

19. Kuroki H, Inomate N, Hamanaka H, Higa K, Chosa E, Tajima N. Predictive factors of Osaka Medical College (OMC) brace treatment in patients with adolescent idiopathic scoliosis. Scoliosis. 2015;10:11.

20. Van Den Bogaart M, Van Royen B, Haanstra T, De Kleuver M, Faraj S. Predictive factors for brace treatment outcome in adolescent idiopathic scoliosis: a best-evidence synthesis. Eur Spine J. 2019;28:511-25. 